\title{
Conhecimento tradicional de pescadores de arraias de água doce da região Amazônica
}

A Etnociência surge como uma importante ferramenta integradora do conhecimento acadêmico e do conhecimento dos pescadores. $O$ objetivo do presente estudo é realizar uma análise do perfil socioeconômico e do conhecimento tradicional sobre as arraias de água doce em relatos de pescadores no município de Barcelos, Amazonas. Foram entrevistados 54 pescadores que foram afetados por acidentes com arraias. Observou-se que $89,0 \%$ são do sexo masculino, demonstrando a manutenção das atividades de pesca que são passadas pelas famílias de crianças e jovens. A proporção de casos ocorreu com pescador com mais de 50 anos $(62,2 \%)$. Há uma predominância do uso de puçá e rapiché que é um tipo de pesca ativa na qual não causa nenhum dano aos animais. Um total de $2,0 \%$ não identificou as cinco espécies presentes no rio Negro. Entre os sintomas apontados pelos pescadores após o acidente, foram citados dor, edema e, algumas vezes, necrose. É possível auxiliar os pescadores de águas doce sobre como se sucedeu os acidentes, as localidades e os mecanismos de atuação dos venenos, bem como a forma com que os pescadores utilizaram para minimizar os efeitos dos ferimentos. As informações geradas aqui podem servir de base para que os ribeirinhos ao ser acidentados por arraias de água doce tenham uma forma de mitigar ou até mesmo de eliminar a dor causada pelo veneno presente nos ferrões desses animais tão temido pelos povos ribeirinhos da Amazônia.

Palavras-chave: Etnoictiologia; Arraias; Água Doce; Pescadores; Amazônia.

\section{Traditional knowledge of fishermen on the sweet freshwater stingrays in the Amazon region}

\begin{abstract}
Ethnoscience emerges as an important tool integrates of academic knowledge and fishermen knowledge. The objective of the present study is analysis of the socioeconomic profile and traditional knowledge about freshwater stingrays from reports of fishermen in the municipality of Barcelos, Amazonas. A total of 54 fishermen were interviewed who were affected by accidents with stingrays. We observed the number of $89.0 \%$ was male, is demonstrates the maintenance of the fishing techniques being passed on by the families the children and young people. The majority of accidents occur with fishermen over 50 years old $(62.2 \%)$. There is predominance of the use hand net which is a type of active fishing in which it does not cause any injury to the animals. According to the traditional knowledge the identification of species from a guide, showed that only $2.0 \%$ didn't identified the five species present in the middle River Negro. Among the symptoms pointed out by the fishermen after the accidents, pain, edema and sometimes necrosis were mentioned. It will be possible to assist fresh fishermen on how accidents, localities and poisoning mechanisms have occurred, as well as how fishermen used them to minimize the effects of the injury. The information generated here can serve as a basis for the riverside to be injured by freshwater stingrays have a way to mitigate or even eliminate the pain caused by the venom present in the stingers of these animals so feared by the people bordering the Amazon.
\end{abstract}

Keywords: Ethnoictiology; Stingrays; Fresh Water; Fishermen; Amazon.

Topic: Uso Sustentável da Biodiversidade

Reviewed anonymously in the process of blind peer.
Received: 02/02/2020

Approved: 10/03/2020
Adriano Teixeira de Oliveira (ID) Instituto Federal do Amazonas, Brasil http://lattes.cnpq.br/9164471794674935 http://orcid org/0000-0003-4988-9878 adriano.oliveira@ifam.edu.br

Daniel da Silva Ladislau (iD

Universidade Estadual do Oeste do Paraná, Brasil http://lattes.cnpq.br/8098824072487689 http://orcid.org/0000-0002-0467-6353 daniel-ladislau@hotmail.com

Maiko Willas Soares Ribeiro (iD Universidade Nilton Lins, Brasil http://lattes.cnpq.br/6299874319353966 http://orcid.org/0000-0002-6155-5646 maikowillas@hotmail.com

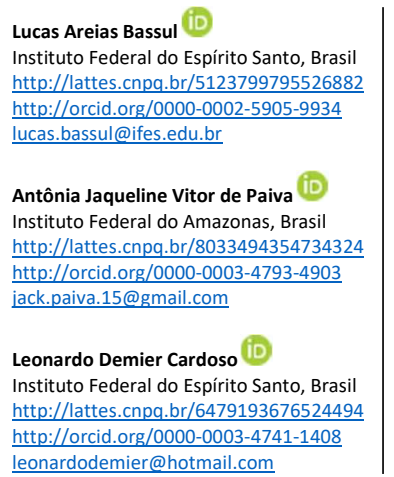

Henrique David Lavander (iD) Instituto Federal do Espírito Santo, Brasil http://lattes cnpq.br/5456683616099165 http://orcid.org/0000-0003-2102-3896 henrique.lavander@ifes.edu.br

Douglas da Cruz Mattos (ID)

Instituto Federal do Espírito Santo, Brasil http://lattes.cnpq.br/0840854277486506 http://orcid.org/0000-0001-7163-4666 douglas uenf@yahoo.com.br

Ariany Rabello da Silva Liebl Instituto Federal do Espírito Santo, Brasil http://lattes.cnpq.br/7556366980080450 http://orcid.org/0000-0002-5010-1294 ny.rabello@gmail.com
Paulo Henrique Rocha Aride (iD) Instituto Federal do Amazonas, Brasil http://lattes.cnpq.br/9087696929404160 http://orcid org/0000-0001-9752-5003 aride@ifam.edu.br

Referencing this:

OLIVEIRA, A. T.; LADISLAU, D. S.; RIBEIRO, M. W. S.; BASSUL, L. A.; PAIVA, A. J. V.; CARDOSO, L. D.; LAVANDER, H. D.; MATTOS, D. C.; LIEBL, A. R. S.; ARIDE, P. H. R.. Conhecimento tradicional de pescadores de arraias de água doce da região Amazônica. Revista Ibero Americana de Ciências Ambientais, v.11, n.2, p.128-135, 2020. DOI: http://doi.org/10.6008/CBPC2179-6858.2020.002.0015 


\section{INTRODUÇÃO}

A literatura demonstra escassez de informações sobre aspectos biológicos de chondrichthyes (peixes cartilaginosos), a partir do conhecimento dos pescadores, o que é denominado de conhecimento tradicional. Geralmente as pesquisas retratam apenas a ocorrência de chondrichthyes com predadores, no entanto, as informações estão concentradas no conhecimento de importância econômica. Em um estudo conduzido por Barbosa-Filho et al. (2014) foram registradas 19 categorias etológicas em relação aos tubarões, em que foram associadas à alimentação, reprodução, comportamentos sociais e migratórios. De acordo com os pescadores do estado da Bahia, é importante saber sobre o comportamento dos tubarões, já que é possível capturá-los, bem como evitar acidentes com este grupo de elasmobrânquios que tradicionalmente é temido pela população.

A região amazônica apresenta um grupo particular de elasmobrânquios, que são as arraias da família Potamotrygonidae, que constituem um grupo único que apresenta representantes no continente Sulamericano (ROSA et al., 2010). Atualmente está família apresenta quatro gêneros, Plesiotrygon, Paratrygon, Potamotrygon e Heliotrygon, sendo registradas 31 espécies (LASSO et al., 2016) e pelo menos 10 espécies ainda não descritas (LUCIFORA et al., 2011).

No Brasil, a exploração de arraias de água doce ainda ocorre exclusivamente de forma extrativa e visa atender a demanda que utiliza arraias de água doce com fins ornamentais (CHAO et al., 2001) e vem crescendo a utilização com fins comestíveis, sendo inclusive observado a vende de filés em supermercados de Manaus (observação pessoal). No Amazonas, o comércio de arraias de água doce com finalidade ornamental é regulado (IBAMA, 2008), na qual 4 espécies podem ser exploradas a partir do sistema de cotas anuais, que limita o tamanho máximo da largura do disco. Atualmente, as espécies Potamotrygon wallacei, Potamotrygon motoro, Potamotrygon schroederi e Potamotrygon orbignyi podem ser comercializadas (IBAMA, 2008). Apesar da importância econômica e das relações tradicionais dos ribeirinhos e pescadores com as arraias de água doce, as informações sobre o conhecimento tradicional desses elasmobrânquios são escassas.

Tradicionalmente, as arraias de água doce têm sua imagem associada com a ocorrência de acidentes, que são causados pela presença de ferrões que contêm toxinas em suas caudas (HADDAD, 2009). Essas espécies de elasmobrânquios são responsáveis pela ocorrência de acidentes dolorosos e alguns casos algumas cicatrizações podem durar meses (HADDAD et al., 2012). Devido a essas características, existe uma aversão por parte dos banhistas, ribeirinhos e pescadores em relação a esse grupo de elasmobrânquios, que normalmente sacrificam os animais e/ou mutilam quando encontram o nos ambientes aquáticos (OLIVEIRA et al., 2015a). O estudo conduzido Reckziegel et al. (2015) observaram que a maioria dos acidentes ocasionados por animais aquáticos no Brasil ocorrerem com arraias $(69,0 \%)$, com altos registro na região norte do Brasil (88,0\%).

Em um estudo conduzido no município Juruá, Amazonas, foi possível observar que a maioria das arraias de água doce são capturadas acidentalmente nas redes, quando a finalidade é a de capturar peixes 
teleósteos a fim de utilizar na alimentação (OLIVEIRA et al., 2015a). De acordo com o conhecimento tradicional dos pescadores da localidade, foi possível observar um total de 9 espécies de arraias de água doce, sendo a espécie predominante a Potamotrygon motoro (OLIVEIRA et al., 2015a), sendo essa confirmando por Rosa et al. (2010) que descreveram que essa espécies apresenta ampla distribuição geográfica. Em São Paulo, aproximadamente 54,0\% dos entrevistados identificaram as arraias Pomotrygon motoro e Potamotrygon falkneri como as arraias que ocasionaram as ferroadas, além disso, foram retratados com sintomas, a dor intolerável e úlceras locais, bem como o uso de imersão em água quente, urina e ervas para controle imediato da dor (HADDAD et al., 2012). Assim, o objetivo do presente trabalho é analisar o perfil sócio econômico e o conhecimento tradicional dos pescadores de arraias que apresentaram acidentes com arraias de água doce do município de Barcelos, Amazonas, médio Rio Negro.

\section{MATERIAIS E MÉTODOS}

O presente estudo foi realizado nas áreas urbana e rural do município de Barcelos, Amazonas, médio Rio Negro. O município de Barcelos é considerado o maior município em extensão territorial do Estado do Amazonas com 112.450, 76 km², localizado a 496 de distância da capital Manaus, com sede a margem direita do Médio Rio Negro, possui uma população de cerca de 26.000 habitantes (IBGE, 2017). Nesta região está localizada a Área de Preservação Ambiental Mariuá, considerada o maior arquipélago fluvial de água doce do mundo (OLIVEIRA et al., 2017a; OLIVEIRA et al., 2015b; MAGRO et al., 2015).

Os dados foram obtidos por meio de entrevistas semiestruturadas com aplicação de questionários contendo perguntas abertas e fechadas, com pescadores de arraias que foram acometidos por acidentes com arraias de água doce $(\mathrm{N}=54)$, as entrevistas ocorreram no período entre janeiro de 2016 a maio de 2019. As perguntas realizadas foram destinadas a obtenção de informações sobre o perfil socioeconômico, aspecto das descrições dos acidentes e as medidas curativas. A média do tempo das entrevistas foi de $\mathbf{4 0}$ minutos de formas individuais, com a devida apresentação do Termo de Consentimento Livre e Esclarecido (TCLE) ao entrevistado, sempre com uma linguagem acessível para devida compreensão do papel da participação pescadores no respectivo estudo. A pesquisa possui autorização junto a Plataforma Brasil (n. 53847316.6.0000.5015). Os dados obtidos foram utilizados para a construção de gráficos e tabelas e apresentados por meio de estatística descritiva com cálculo de frequência relativa.

\section{RESULTADOS E DISCUSSÃO}

Dos entrevistados um total de $89,0 \%$ foram do sexo masculino, indicando predominância da atividade de pesca por homens. Por outro lado, um percentual de $11,0 \%$ dos pescadores entrevistados era do sexo feminino, indicando que a atividade pesqueira de arraias de água doce para fins ornamentais também é composta por mulheres, como Chao et al. (2001) também descreveu nas pescarias de peixes ornamentais no médio rio Negro, Amazonas. Em relação às localidades em que vivem os pescadores que foram afetados por acidentes com arraias, a Tabela 1 apresenta as características, em que foram observadas 
uma maior incidência de pescadores que vivem na área urbana do município de Barcelos, indicando que esses pescadores são os únicos que interagem mais acidentalmente com arraias de água doce. Esses resultados demonstram resultados semelhantes aos relatados por Oliveira et al. (2015a) ao investigar essa relação com pescadores do município de Juruá, Amazonas.

Tabela 1: Localidade residencial dos pescadores acidentados com arraias de água doce do médio Rio Negro, Amazonas.

\begin{tabular}{|l|l|}
\hline Comunidades & Percentual \\
\hline Área urbana de Barcelos & 73,0 \\
\hline Bacabal & 7,0 \\
\hline Mariuá & 4,0 \\
\hline Marione & 2,0 \\
\hline Aracá & 2,0 \\
\hline Cumarú & 2,0 \\
\hline Maricá & 2,0 \\
\hline Bacuri & 2,0 \\
\hline São Lázaro & 2,0 \\
\hline
\end{tabular}

Na região amazônica é notória a inserção de jovens nas atividades pesqueiras. No presente estudo foi registrado que $2,0 \%$ dos pesquisados são menores de idade. A Tabela 2 apresenta os resultados do perfil etário dos pescadores do médio rio Negro, Amazonas. No qual demonstra que a maioria dos acidentes ocorrem com pescadores com mais de 50 anos de idade (62,2\%), indicando que os acidentes podem estar relacionado com a diminuição da acuidade visual devido à idade, cansaço nessa faixa etária é mais recorrente ou mesmo o excesso de confiança desses pescadores, porque eles têm uma vasta experiência no conhecimento da localidade. $\mathrm{O}$ vasto conhecimento biológico que eles têm sobre esses elasmobrânquios ou o manuseio. Estes resultados são diferentes dos resultados encontrados no município de Juruá, Amazonas, que indicaram uma faixa etária predominante entre 30 e 40 anos de idade (OLIVEIRA et al., 2015a).

Tabela 2: Perfil de idade dos pescadores afetados por acidentes com arraias de água doce no médio Rio Negro, Amazonas.

\begin{tabular}{|l|l|}
\hline Idade & Percentual (\%) \\
\hline Menor de 17 anos de idade & 2,0 \\
\hline 18 a 19 anos de idade & 0,0 \\
\hline 20 a 29 anos de idade & 11,2 \\
\hline 30 a 39 anos de idade & 2,0 \\
\hline 40 a 49 anos de idade & 22,6 \\
\hline 50 a 59 anos de idade & 35,8 \\
\hline Mais de 60 anos de idade & 26,4 \\
\hline
\end{tabular}

A Tabela 3 apresenta o perfil educacional dos pescadores, é evidente que a maioria $(83,3 \%)$ é analfabeta ou tem ensino fundamental incompleto,, essa característica também foi encontrada por Oliveira et al. (2015a) que encontraram percentual de $94,0 \%$.

Tabela 3: Perfil escolar de pescadores acometidos por acidentes com arraias de água doce do médio rio Negro.

\begin{tabular}{|l|l|}
\hline Nível escolar & Percentual (\%) \\
\hline Analfabeto & 28,3 \\
\hline Ensino Fundamental Incompleto & 54,6 \\
\hline Ensino Fundamental Completo & 5,7 \\
\hline Ensino Médio Incompleto & 9,4 \\
\hline Ensino Médio Completo & 2,0 \\
\hline
\end{tabular}

Em relação ao tempo em que os pescadores residem nas comunidades, a predominância foi entre 20 
a 30 anos, sendo indicada pelos pescadores, conforme apresentado na Figura 1. No estudo realizado no rio Juruá houve predomínio do tempo de residência entre 30 e 40 anos (OLIVEIRA et al., 2015a), sendo portanto, diferente do presente estudo.

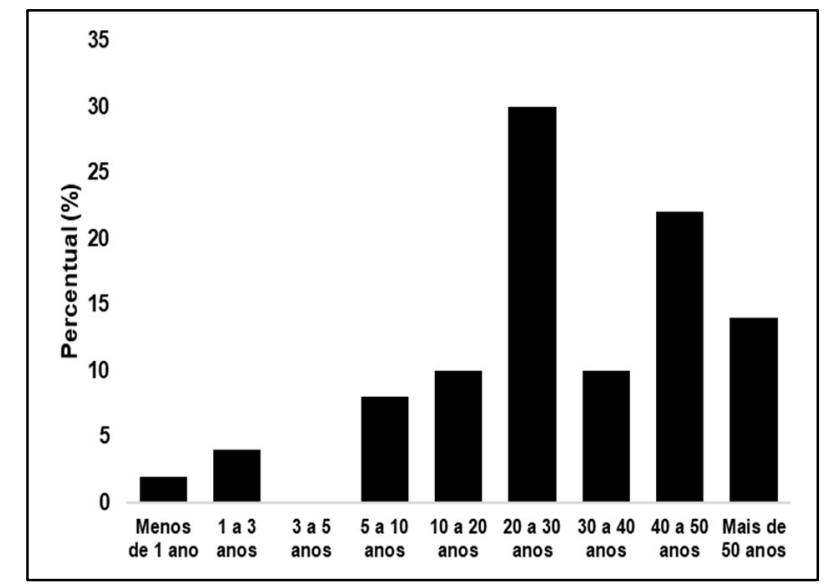

Figure 1: Tempo de vida dos pescadores nas comunidades.

A Figura 2 apresenta as ferramentas utilizadas pelos pescadores do médio rio Negro para capturar arraias de água doce. Existe predominância do uso de rede de mão (rapiché ou puçá) que é um tipo de pesca ativa na qual não causam nenhum dano aos animais, sendo o instrumento recomendado para a captura de peixes ornamentais. Os resultados do presente estudo são semelhantes às formas de capturas das arraias de água doce, descritas por Oliveira et al. (2012), Oliveira et al. (2016) e Oliveira et al. (2017b). Conforme descrito pelos pescadores, a captura de arraias com outros instrumentos (Figura 2), além do rapiché, ocorre de forma não intencional.

Na Amazônia brasileira, existe a maior diversidade de arraias de água doce (LASSO et al. 2016). De acordo com o conhecimento tradicional dos pescadores, a identificação das espécies de arraias de água doce da localidade a partir de um guia, demonstra que apenas 2,0\% dos entrevistados (Tabela 4) não souberam identificar, visto que na localidade pesquisada são registradas 5 espécies (LASSO et al., 2016). Desta forma, é possível afirmar que os pescadores que tiveram relatos de acidentes com arraias possuem um conhecimento marcante sobre as espécies existentes na localidade.

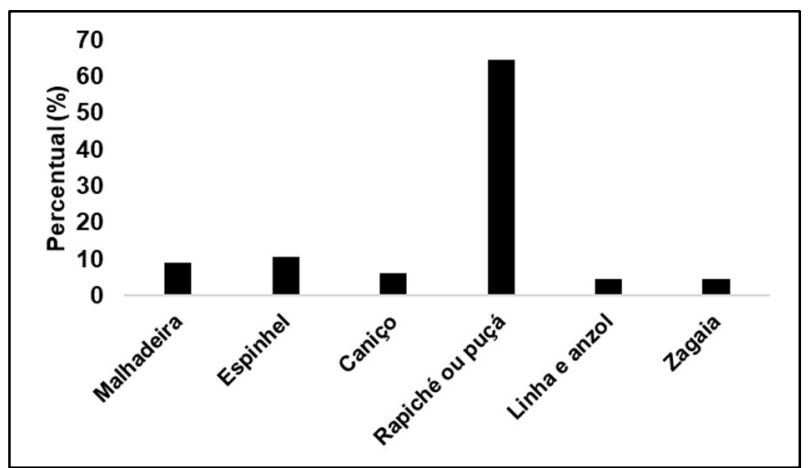

Figure 2: Instrumentos de pesca utilizados para capturar arraias de água doce no médio rio Negro, Amazonas.

Segundo o conhecimento tradicional dos pescadores, é possível afirmar que as arraias de água doce têm preferência pelas áreas de praia e pelo rio, no entanto, outros três habitats foram descritos (Figura 3). 
No estudo conduzido por Oliveira et al. (2015a) as arraias de água doce possuem preferências por lagos no município de Juruá, Amazonas.

Tabela 4: Espécies de arraias de água doce identificadas por pescadores que foram acometidos por acidentes no médio rio Negro.

\begin{tabular}{|l|l|}
\hline Espécies & Percentual (\%) \\
\hline Potamotrygon wallacei & 27,8 \\
\hline Potamotrygon motoro & 29,7 \\
\hline Potamotrygon orbignyi & 10,1 \\
\hline Potamotrygon schroederi & 16,5 \\
\hline Paratrygon aiereba & 13,3 \\
\hline Outras & 2,5 \\
\hline
\end{tabular}

O período de maior ocorrência das arraias de água doce é o de águas baixas (vazante e seco), na qual os períodos podem variar entre os meses de agosto a abril, essa característica já foi descrita por Duncan et al. (2010). Segundo relatos dos pescadores, quando houve captura das arraias eles foram devolvidos a natureza $(32,0 \%)$, foram mortos $(23,0 \%)$ ou mutilados $(22,0 \%)$, indicando altas taxas de pesca negativa $(45,0 \%)$ na localidade. Em um estudo realizado no rio Juruá se estimou que em 2012 houve o sacrifício de 10.660 arraias, mutilação de 3.562 e a liberação de apenas 286 arraias (OLIVEIRA et al., 2015a). Estes resultados demonstram o quanto esses elasmobrânquios amazônicos são temidos na localidade, como demonstrado pelos pescadores do médio rio Negro que afirmaram $(80,0 \%)$ que os memos são perigosos.

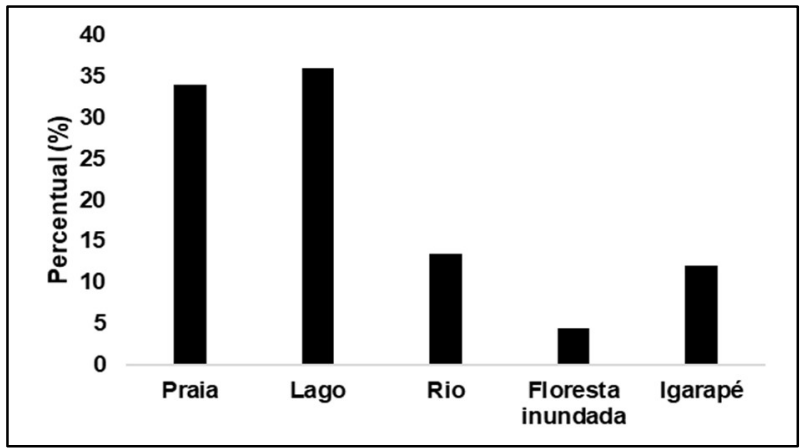

Figure 3: Preferência de habitat das arraias de água doce de acordo com o conhecimento tradicional de pescadores do médio Rio Negro, Amazonas.

É evidente que os pés $(73,8 \%)$ são os mais afetados, resultados também encontrados por Haddad (2009), Haddad et al. (2012) e Oliveira et al. (2015a). Os ferimentos ocasionados ocorreram principalmente quando os pescadores puxavam as canoas, trocavam as águas das caçapas contendo as arraias e quando pescavam. Também foram registrados acidentes nas mãos $(16,9 \%)$, pernas $(4,6 \%)$, coxas $(3,1 \%)$ e abdomen $(1,5 \%)$.

Entre os sintomas assinalados pelos pescadores após os acidentes, foram descritos dor, inchaço e, por vezes, necrose, essas características também foram registradas por Haddad (2009), Haddad et al. (2012) e Oliveira et al. (2015a). De fato, há uma relação de contato frequente entre pescadores e arraias de água doce. Quando perguntado sobre o número de vezes que eles foram afetados por ferroadas, um total de $37,5 \%$ afirmaram terem apenas um acidente, $21,9 \%$ duas vezes, $21,9 \%$ três vezes, $6,3 \%$ quatro vezes, $3,1 \%$ cinco vezes, $3,1 \%$ seis vezes, $3,1 \%$ dez vezes e $3,1 \%$ vinte e duas vezes. De acordo com o relatório dos 
pescadores que tinham sido ferroados, houve registro de dor insuportável até a existência de apenas dormência da localidade acometida.

Os pescadores possuem baixo nível de escolaridade ou conhecimento sobre medidas curativas para acidentes causados por arraias de água doce (HADDAD et al., 2012). Quando eles enfrentaram essa situação, existe uma série de procedimentos que eles realizam. Aqui vamos mencionar algumas das medidas que foram registradas durante as entrevistas com pescadores do médio rio Negro: Dipirona, anestésicos, água morna, água quente com sal, água quente com sabão, nada, açúcar queimado, água em temperatura ambiente, ovo de galinha cozido, mingau de aveia quente e povidine como as remediações mais descritas. De fato, o aumento da temperatura parece ser conhecido pelos pescadores como uma contramedida eficaz contra a toxina presente nos ferrões das arraias de água doce. Essa medida foi indicada por Haddad et al. (2012) como sendo um dos mecanismos mais eficientes para reduzir a dor imediatamente devido à natureza termolábil das toxinas.

Outras medidas indicadas pelos pescadores curativas foram a utilização de gasolina, pilha, lama, fezes de minhoca e saliva. Na verdade, estas medidas podem agravar as infecções no edema, como afirma Haddad et al. (2012) e Oliveira et al. (2015a). Quanto ao uso de ervas no edema, o uso de cipó defumado e casca de madeira de florestal inundada foram apontados pelos pescadores.

De acordo com os pescadores as arraias são animais que utilizam os ferrões como forma de defesa, quando esses perdem os ferrões eles têm como características ficarem dóceis. Sobre a importância das arraias, foi descrita por um dos pescadores que eles limpam o fundo do rio, sendo assim importante na ciclagem de nutrientes da cadeia alimentar, e também há relatos de que eles também se alimentam de insetos, camarões e peixes, como descrito por Shibuya et al. (2009) sobre três espécies de arraias do médio rio Negro. Tem sido relatado que o local onde as arraias se encontram (popularmente chamado de cama das arraias) é onde outras espécies de peixes, como a Cichla spp. tucunarés, depositam seus ovos. Esse tipo de informação pode ser usado para a exploração ordenada do uso das arraias para fins comerciais. Também foi descrito que deveria haver uma maior limitação da largura do disco para fins de exploração ornamental atualmente utilizado, aumentando assim o tamanho dos animais utilizados no mercado de aquários.

\section{CONCLUSÕES}

A partir do conhecimento dos pescadores que foram ferroados por arraias de água doce é possível auxiliar os pescadores de águas doces sobre como se sucedeu os acidentes, as localidades e os mecanismos de atuação dos venenos, bem como a forma com que os pescadores utilizaram para minimizar os efeitos do ferimento. As informações geradas aqui podem servir de base para que os ribeirinhos ao ser acidentados por arraias de água doce tenham uma forma de mitigar a dor causada pelo veneno presente nos ferrões desses animais tão temido pelos povos ribeirinhos da Amazônia.

AGRADECIMENTOS: à Fundação de Amparo à Pesquisa do Estado do Amazonas (FAPEAM), processo $\mathrm{n}$. 
$062.00933 / 2015$.

\section{REFERÊNCIAS}

BARBOSA-FILHO, M. L. V.; SCHIAVETTI, A.; ALARCON, D. T.; COSTA-NETO, E. M.. 'Shark is the man!': ethnoknowledge of Brazil's South Bahia fishermen regarding shark behaviors. Journal of ethnobiology and ethnomedicine, v.10, n.54, 2014. DOI: http://doi.org/10.1186/1746-4269-10$\underline{54}$

CHAO, N. L.; PRANG, G.. Project Piaba- towards a sustainable ornamental fishery in the Amazon. Aquarium Sciences and Conservation, n.1, v.2, p.105-111, 1997.

DUNCAN, W. P.; INOMATA, S. O.; FERNANDES, M. S.. Comércio de raias de água doce na região do médio Rio Negro, estado do Amazonas, Brasil. Revista Brasileira de Engenharia de Pesca, v.5, p.19-22, 2010.

HADDAD, V.. Acidentes por animais peçonhentos. In: ARRUDA, M. M.; CARRILHO, F. J.; ALVES, V. A. F.; CASTILHO, E. A.; CERRI, G.; WEN C. L.. Clínica Médica. São Paulo: Manole, 2009. p.553-613.

HADDAD, V.; JUNIOR, F.; LUIZ, E.; RIBEIRO, F. A. H.; ANCHESCHI, B. C.; CASTRO, G. I. P.; MARTINS, R. C.; PAZUELO, G. B.; FUJII, J. R.; VIEIRA, R. B.; GARRONE-NETO, D.. Trauma and Envenoming Caused by Stingrays and Other Fish in a Fishing Community in Pontal do Paranapanema, State of São Paulo, Brazil: Epidemiology, Clinical Aspects, and Therapeutic and Preventive Measures. Revista da Sociedade Brasileira de Medicina Tropical, v.45, p.238-242, 2012. DOI: http://doi.org/10.1590/S0037-86822012000200019

IBAMA. Instituto Brasileiro do Meio Ambiente e dos Recursos Naturais Renováveis. Instrução Normativa №. 204/2008. IBAMA, 2008.

IBGE. Instituto Brasileiro de Geografia e Estatística. Censo demográfico. Rio de Janeiro: IBGE, 2017.

LASSO, C. A.; ROSA, R. S.; MORALES-BETANCOURT, M. A.; GARRONE-NETO, D.; CARVALHO, M.. Rayas de agua dulce (Potamotrygonidae) de Suramérica. Parte II: Colombia, Brasil, Perú, Bolivia, Paraguay, Uruguay y Argentina. Bogotá: Colombia, 2016.

LUCIFORA, L. O.; GARCÍA, V. B.; WORM, B.. Global diversity hotspots and conservation priorities for sharks. Plos One, v.6, n.5, 2011. DOI:

http://doi.org/10.1371/journal.pone.0019356

MAGRO, N. M.; OLIVEIRA, A. T.; DAVIES, A.; ODWYER, L. H.. First report and description of a Cyrilia sp. (Apicomplexa: Haemogregarinidae) from a freshwater Cururu Stingray Potamotrygon cf. histrix (Elasmobranchii: Potamotrygonidae), from the Amazon Region, Brazil. Journal of Fish Diseases, v.38, p.1-5, 2015. DOI:

https://doi.org/10.1111/ifd.12425
OLIVEIRA, A. T.; ARAUJO, M. L. G.; PANTOJA, J.; ARIDE, P. H. R.. Ecophysiological interactions and water-related physicochemical parameters among freshwater stingrays. Brazilian Journal of Biology, v.77, p.616-621, 2017b. DOI: http://doi.org/0.1590/1519-6984.01816

OLIVEIRA, A. T.; ARAÚJO, M. L. G.; PANTOJA-LIMA, J.; ARIDE, P. H. R.; TAVARES-DIAS, M.; BRINN, R. P.; MARCON, J. L.. Cyrilia sp. (Apicomplexa: Haemogregarinidae) in the Amazonian freshwater stingray Potamotrygon wallacei (cururu stingray) in different hydrological phases of the Rio Negro. Brazilian Journal of Biology, v.77, p.413-416, 2017a. DOI: http://dx.doi.org/10.1590/1519-6984.00416

OLIVEIRA, A. T.; LEMOS, J. R. G.; SANTOS, M. Q. C.; ARAUJO, M. L. G.; TAVARES-DIAS, M.; MARCON, J. L.. Procedimentos de manuseio e de colheita de sangue em arraias de água doce. Boletim da EMBRAPA, v.77, p.1-18, 2012.

OLIVEIRA, A. T.; LIMA, E. C.; SANTOS, S. M.; ARAUJO, R. L.; PANTOJA-LIMA, J.; ROCHA, P. H. A.. Relação entre as populações naturais de arraias de água doce (Myliobatiformes: Potamotrygonidae) e pescadores no baixo Rio Juruá, estado do Amazonas, Brasil. Biota Amazônia, v.5, p.108-111, 2015a. DOI: http://dx.doi.org/10.18561/21795746/biotaamazonia.v5n3p108-111

OLIVEIRA, A. T.; SANTOS, M. Q. C.; ARAUJO, M. L. G.; LEMOS, J. R. G.; SALES, R. S. A.; PANTOJA-LIMA, J.; TAVARES-DIAS, M.; MARCON, J. L.. Hematological parameters of three freshwater stingray species (Chondrichthyes:

Potamotrygonidae) in the middle Rio Negro, Amazonas state. Biochemical Systematics and Ecology, v.69, p.33-40, 2016. DOI: http://doi.org/10.1016/i.bse.2016.07.002

OLIVEIRA, A. T.; SANTOS, M. Q. C.; LEMOS, J. R. G.; TAVARESDIAS, M.; MARCON, J. L. . Comparison of the Effects of Anticoagulants Used in Blood Collection to Determine Blood Parameters of Free-Living Stingrays from the Potamotrygon genus (Elasmobranchii: Potamotrygonidae). Biota Amazônia, v.5, p.55-58, 2015b. DOI: http://dx.doi.org/10.18561/21795746/biotaamazonia.v5n3p55-58

ROSA, R. S.; CHARVET-ALMEIDA, P.; QUIJADA, C. C. D.. Biology of the South American Potamotrygonid Stingrays. In: CARRIER, J. F.; MUSICK, J. A.; HEITHAUS, M. R.. Sharks and their relatives II: biodiversity, adaptive physiology, and conservation. CRC Press, 2010. P.241-286.

SHIBUYA, A.; ARAUJO, L.; ZUANON, J.. Analysis of stomach contents of freshwater stingrays (Elasmobranchii, Potamotrygonidae) from the middle Negro River, Amazonas, Brazil. Pan-American Journal Of Aquatic Sciences, v.4, p.466-475, 2009.

A CBPC - Companhia Brasileira de Produção Científica (CNPJ: 11.221.422/0001-03) detém os direitos materiais desta publicação. Os direitos referem-se à publicação do trabalho em qualquer parte do mundo, incluindo os direitos às renovaç̃os, expansões e disseminacõos da contribuiç̃o, bem como outros direitos subsidiários. Todos os trabalhos publicados eletronicamente poderão posteriormente ser publicados em coletâneas impressas sob coordenação da Sustenere Publishing, da Companhia Brasileira de Produção Científica e seus parceiros autorizados. Os (as) autores (as) preservam os direitos autorais, mas não têm permissão para a publicação da contribuição em outro meio, impresso ou digital, em português ou em tradução. 